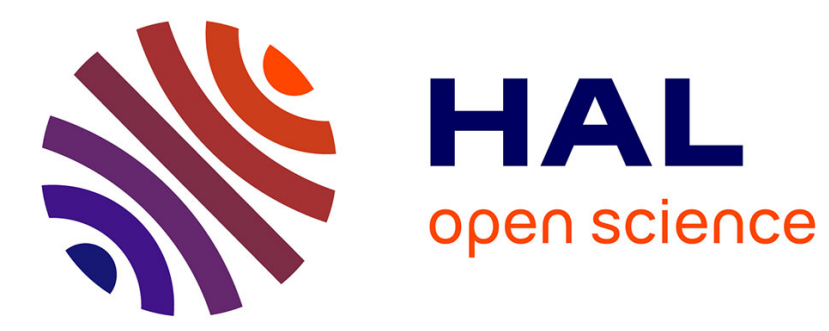

\title{
A new form of the elliptic relaxation equation to account for wall effects in RANS modeling
}

Remi Manceau, Kemal Hanjalić

\section{To cite this version:}

Remi Manceau, Kemal Hanjalić. A new form of the elliptic relaxation equation to account for wall effects in RANS modeling. Physics of Fluids, 2000, 12 (9), pp.2345-2351. 10.1063/1.1287517 . hal02991003

\section{HAL Id: hal-02991003 \\ https://hal.science/hal-02991003}

Submitted on 5 Nov 2020

HAL is a multi-disciplinary open access archive for the deposit and dissemination of scientific research documents, whether they are published or not. The documents may come from teaching and research institutions in France or abroad, or from public or private research centers.
L'archive ouverte pluridisciplinaire HAL, est destinée au dépôt et à la diffusion de documents scientifiques de niveau recherche, publiés ou non, émanant des établissements d'enseignement et de recherche français ou étrangers, des laboratoires publics ou privés. 


\title{
A new form of the elliptic relaxation equation to account for wall effects in RANS modeling
}

\author{
Rémi Manceau and Kemal Hanjalića) \\ Department of Applied Physics, Thermofluids Section, Delft University of Technology, Lorentzweg 1, \\ P.O. Box 5046, 2600 GA, Delft, The Netherlands
}

(Received 12 January 2000; accepted 5 June 2000)

\begin{abstract}
Different methods for improving the behavior in the logarithmic layer of the elliptic relaxation equation, which enable the extension of Reynolds stress models or eddy viscosity models down to the wall, are tested in a channel flow at $\operatorname{Re}_{\tau}=590$ and compared with direct numerical simulation (DNS) data. First, a priori tests are performed in order to confirm the improvement predicted by the theory, either with the Rotta+IP (isotropization of production) model or the Speziale-SarkarGatski (SSG) model as the source term of the elliptic relaxation equation. The best form of the model is then used for full simulations, in Durbin second moment closure or in the frame of the $\overline{v^{2}}-f$ model. It is shown that the results can be significantly improved, in particular by using a formulation based on the refinement of the modeling of the two-point correlations involved in the redistribution term. (C) 2000 American Institute of Physics. [S1070-6631(00)50709-0]
\end{abstract}

\section{INTRODUCTION}

One of the most important unclosed terms appearing in the Reynolds stress transport equation is the redistributive term arising from pressure fluctuations. Almost all models for this term used so far are based on the pioneering propositions of Chou ${ }^{1,2}$ and Rotta, ${ }^{3}$ which consist in expressing the redistribution as an algebraic expression of mean quantities of the flow. This approach was originally introduced for high-Reynolds number regions, and is based on the quasihomogeneity and locality assumptions that are only valid "not too close to solid boundaries." 1 In order to extend models down to the wall, damping functions or nonlinear terms are usually introduced. However, seeking to reproduce the nearwall behavior of turbulence by introducing complex correction terms appears somewhat inconsistent when the basic assumptions are not valid in this region. ${ }^{4,5}$ On the contrary, the elliptic relaxation approach ${ }^{6,7}$ is based on a theoretical analysis and a simple modeling of the two-point correlations involved in the integral form of the redistribution term, and does not use the previously quoted Chou's assumptions. Models based on this approach, and in particular the $\overline{v^{2}}-f$ model, ${ }^{6}$ reduced to three transport equations, have been successfully applied in a number of different situations. ${ }^{8-13}$

Wizman et al. ${ }^{14}$ emphasized that the behavior of the elliptic relaxation operator in the logarithmic layer is not fully satisfactory, since it induces an amplification of the redistribution. Indeed, in this region, the total damping of the redistribution is the result of a balance between two phenomena. The first one is the reflection of the pressure fluctuations by the solid wall, which, contrary to the common belief, enhances the pressure scrambling effects that tend to make the turbulence more isotropic. ${ }^{15}$ However, this effect is much

\footnotetext{
a) Author to whom correspondence should be addressed. Telephone: (31) 15278 1735; Fax: (31) 15278 1204; Electronic mail: hanjalic@ws.tn.tudelft.nl
}

weaker than the second one: the blocking of the velocity fluctuations normal to the wall due to wall impermeability, which enforces a splatting of the near wall eddy structure, making the turbulence field highly anisotropic and forcing it to approach the two-component limit. It is this blockage effect, which is of an elliptic nature, that is assumed to be modeled by the elliptic relaxation approach.

In order to correct this shortcoming of the model, Wizman et al. proposed different new formulations of the operator, using arbitrary corrections. Manceau et al. ${ }^{15}$ used a different route to reach the same purpose: they showed, by a direct numerical simulation (DNS) channel flow database analysis, that the spurious behavior of the operator is due to the intuitive assumptions used by Durbin ${ }^{6,7}$ which do not account for the asymmetry of the two-point correlations involved in the integral equation of the redistribution term. Using an asymmetric correlation function, they derived a new formulation of the operator, which does not exhibit the same amplification as the original one.

The purpose of this work is to confirm this theoretical result by computations. A priori tests are first performed, in order to determine the effect of the elliptic operator on the source term [Rotta+IP (isotropization of production) or Speziale-Sarkar-Gatski (SSG) model] for different forms of this operator. Full simulations in a channel are then performed with the formulations proposed by Wizman et al. and Manceau et al., either in the frame of a second moment closure or of the $\overline{v^{2}}-f$ model. These computations allow the evaluation of the improvement due to the elimination of the spurious amplification exhibited by the original model.

\section{THEORETICAL BACKGROUND}

\section{A. The original model}

The redistributive term that appears in the Reynolds stress transport equation is 


$$
\rho \phi_{i j}^{*}=-\overline{u_{i} \frac{\partial p}{\partial x_{j}}}-\overline{u_{j} \frac{\partial p}{\partial x_{i}}} .
$$

Using the integral solution of the Poisson equation satisfied by the fluctuating pressure, this term can be expressed as

$$
\rho \phi_{i j}^{*}(\mathbf{x})=\int_{\Omega} \Psi_{i j}\left(\mathbf{x}, \mathbf{x}^{\prime}\right) G_{\Omega}\left(\mathbf{x}, \mathbf{x}^{\prime}\right) d V\left(\mathbf{x}^{\prime}\right),
$$

where

$$
\Psi_{i j}\left(\mathbf{x}, \mathbf{x}^{\prime}\right)=-\overline{u_{i}(\mathbf{x}) \nabla^{2} \frac{\partial p}{\partial x_{j}}\left(\mathbf{x}^{\prime}\right)}-\overline{u_{j}(\mathbf{x}) \nabla^{2} \frac{\partial p}{\partial x_{i}}\left(\mathbf{x}^{\prime}\right),}
$$

and $G_{\Omega}$ is the Green function of the domain. Durbin ${ }^{6}$ proposed to define the correlation function $f\left(\mathbf{x}, \mathbf{x}^{\prime}\right)$ by

$$
\Psi_{i j}\left(\mathbf{x}, \mathbf{x}^{\prime}\right)=\Psi_{i j}\left(\mathbf{x}^{\prime}, \mathbf{x}^{\prime}\right) f\left(\mathbf{x}, \mathbf{x}^{\prime}\right),
$$

and to model it by a simple isotropic exponential function

$$
f\left(\mathbf{x}, \mathbf{x}^{\prime}\right)=\exp \left(-\frac{r}{L}\right)
$$

where $r=\left\|\mathbf{x}^{\prime}-\mathbf{x}\right\|$ and $L$ is the correlation length scale.

Using the model (5) and assuming that the Green function can be approximated by the free-space Green function, ${ }^{16}$ Eq. (2) becomes

$$
\rho \phi_{i j}^{*}(\mathbf{x})=-\int_{\Omega} \Psi_{i j}\left(\mathbf{x}^{\prime}, \mathbf{x}^{\prime}\right) \frac{\exp (-r / L)}{4 \pi r} d V\left(\mathbf{x}^{\prime}\right) .
$$

This expression involves $\exp (-r / L) / 4 \pi r$, which is the freespace Green function associated with the operator $-\nabla^{2}$ $+1 / L^{2}$. Thus, Eq. (6) can be inverted to give

$$
\phi_{i j}^{*}(\mathbf{x})-L^{2} \nabla^{2} \phi_{i j}^{*}(\mathbf{x})=-\frac{L^{2}}{\rho} \Psi_{i j}(\mathbf{x}, \mathbf{x}) .
$$

Noting that in homogeneous situations, the second term on the left-hand side vanishes, the source term can be replaced by any quasihomogeneous model $\phi_{i j}^{h}$, and Eq. (7) becomes

$$
\phi_{i j}^{*}-L^{2} \nabla^{2} \phi_{i j}^{*}=\phi_{i j}^{h},
$$

which is called elliptic relaxation equation. The length scale $L$ is modeled usually by $C_{L} k^{3 / 2} / \varepsilon$ but bounded by the Kolmogorov length scale $C_{L} C_{\eta} \nu^{3 / 4} / \varepsilon^{1 / 4}$ in order to avoid a singularity at the wall (and also to reproduce the real behavior of the correlation length scale observed in DNS results). ${ }^{15}$

The specificity of this model is that the redistributive term is not given by an algebraic expression, but by a differential equation, which preserves the nonlocal character of this term. This enables the reproduction of the wall-blocking effect, provided that the correct boundary conditions are applied, ${ }^{7}$ which requires the resolution of the elliptic relaxation equation for the function $f_{i j}=\phi_{i j}^{*} / k$ instead of $\phi_{i j}^{*}$ :

$$
f_{i j}-L^{2} \nabla^{2} f_{i j}=f_{i j}^{h},
$$

where $f_{i j}^{h}=\phi_{i j}^{h} / k$.

The effect of the elliptic relaxation equation is two-fold.

It enforces the correct damping of the redistribution at the wall, because of its boundary conditions. The latter are chosen in order that the redistribution balances the difference between the viscous diffusion and the dissipation in the vicinity of the wall. This enables the reproduction of the correct asymptotic behaviors of the different components of the Reynolds stress and of the two-component limit of turbulence.

(ii) In a part of the logarithmic region, too far from the wall to be under the influence of the boundary conditions, the redistribution is amplified, as emphasized by Wizman et al. ${ }^{14}$ Indeed, all quasihomogeneous models $\phi_{i j}^{h}$ basically behave as $y^{-1}$ in this region, so that using the standard logarithmic layer values $k$ $=u_{\tau}^{2} / C_{\mu}^{1 / 2}$ and $\varepsilon=u_{\tau}^{3} / \kappa y$, the solution of Eq. (9) is $f_{i j}=\Gamma f_{i j}^{h}$, with the amplification factor $\Gamma \simeq 1.51$. Note that this result is obtained assuming that the solution is not affected by the boundary conditions.

Thus, the overall benefit obtained from the use of the elliptic relaxation equation is due to its boundary conditions that are "elliptically relaxed" up to the logarithmic layer. The amplification arising from the elliptic operator is a side effect that penalizes the predictions in the upper part of the logarithmic layer. The purpose of the next section is to show how this shortcoming can be corrected.

\section{B. Correction of the logarithmic layer behavior}

Manceau et al. ${ }^{15}$ showed that this behavior is a consequence of the fact that the anisotropy of the correlation function $f\left(\mathbf{x}, \mathbf{x}^{\prime}\right)$, and, in particular, its asymmetry in the wallnormal direction due to the strong inhomogeneity in the vicinity of the wall, is not accounted for by the model Eq. (5). They proposed to use the gradient of the length scale to identify the main direction of inhomogeneity, in the following manner:

$$
f\left(\mathbf{x}, \mathbf{x}^{\prime}\right)=\exp \left(-\frac{r}{L+\beta\left(\mathbf{x}^{\prime}-\mathbf{x}\right) \cdot \boldsymbol{\nabla} L}\right) .
$$

Considering the new term as a small correction and using a Taylor series expansion, a new form of the elliptic relaxation equation can be derived:

$$
\left(1+16 \beta(\nabla L)^{2}\right) f_{i j}-L^{2} \nabla^{2} f_{i j}-8 \beta L \nabla L \cdot \nabla f_{i j}=f_{i j}^{h} .
$$

The amplification factor $\Gamma$, obtained by the same method as used in Sec. IA, is, for this formulation,

$$
\Gamma=\frac{1}{1+2(12 \beta-1) C_{L}^{2} C_{\mu}^{-3 / 2} \kappa^{2}} .
$$

The coefficient $\beta$, which determines the amount of asymmetry introduced in the model, can then be adapted to obtain different effects, depending on the quasihomogeneous model used as the source term. For instance, with the SSG model, which predicts correctly the redistribution in the logarithmic layer, $\beta=1 / 12$ can be chosen, in order to obtain a neutral model $(\Gamma=1)$. Note that neutral means only here that the operator is neutral, i.e., that the previously quoted "side effect' is not exhibited; in this case, the effect of the elliptic relaxation equation is only due to its boundary conditions. With the Rotta+IP model, the overestimation of the redistri- 
TABLE I. Summary of the logarithmic layer behavior of different formulations of the elliptic relaxation equation.

\begin{tabular}{lccc}
\hline \hline Model & \multicolumn{1}{c}{ Equation } & $\gamma$ & $\Gamma$ \\
\hline$D^{\mathrm{a}}$ & $f_{i j}-L^{2} \nabla^{2} f_{i j}=f_{i j}^{h}$ & -2 & 1.51 \\
$W 1^{\mathrm{b}}$ & $f_{i j}-\nabla^{2}\left(L^{2} f_{i j}\right)=f_{i j}^{h}$ & 0 & 1 \\
$W 2^{\mathrm{b}}$ & $f_{i j}-L^{2} \nabla \cdot\left(\frac{1}{L^{2}} \nabla\left(L^{2} f_{i j}\right)\right)=f_{i j}^{h}$ & 2 & 0.75 \\
$M^{\mathrm{c}}$ & $\left(1+16 \beta(\nabla L)^{2}\right) f_{i j}-L^{2} \nabla^{2} f_{i j}$ & $2(12 \beta-1)$ & {$[0 ; 1.51]$} \\
& $-8 \beta L \nabla L \cdot \nabla f_{i j}=f_{i j}^{h}$ & & \\
\hline \hline
\end{tabular}

${ }^{\text {aReference } 6 .}$.

${ }^{\mathrm{b}}$ Reference 14 .

'Reference 15 .

bution can be corrected by choosing a coefficient $\beta$ greater than 1/12. In Table I are summarized the coefficients $\gamma$ given by different formulations of the elliptic relaxation equation, $\gamma$ being defined by

$$
\Gamma=\frac{1}{1+\gamma C_{L}^{2} C_{\mu}^{-3 / 2} \kappa^{2}} .
$$

The numerical value of $\Gamma$ is also given, with $C_{L}$ chosen such that $L=\kappa y$ in the logarithmic layer $\left(C_{L}=C_{\mu}^{3 / 4}\right)$. Concerning the $M$ formulation, an interval of amplification factors is given, since the coefficient $\beta$ can be chosen between zero and infinity.

It must be emphasized that these theoretical results are obtained by a simple analysis that does not account for boundary conditions, and is accordingly only valid in the infinite Reynolds number limit. The aim of the next section is to investigate numerically this behavior at finite Reynolds number.

\section{A PRIORI TESTS}

The DNS database ${ }^{17}$ for a channel flow at $\operatorname{Re}_{\tau}=590$ is used to perform a priori tests of the different formulations of the elliptic relaxation equation presented in Table I. These tests consist in solving the equations given in Table I with the terms $f_{i j}^{h}$ and $L$ taken from the DNS database. The exact boundary condition $f_{i j}=f_{i j}^{\text {DNS }}$ is also applied at the wall. The equation is solved in only one-half of the channel, a symmetry boundary condition being used at the center.

Results obtained for $f_{22}$, with the Rotta + IP model as the source term $\phi_{22}^{h}$, are shown multiplied by $k\left(\phi_{22}^{*}\right.$ $\left.=k f_{22}\right)$ in Fig. 1(a). First, it can be observed that the Rotta+IP model, without the elliptic relaxation correction, overestimates the redistribution in the logarithmic layer and gives totally wrong results in the buffer layer and the viscous sublayer. In order to fairly compare the different formulations, the coefficient $C_{L}$ is tuned in each case to correctly predict the amplitude of the peak of $\phi_{22}^{*}$. It can be seen that the application of the original form of the elliptic relaxation equation (model $D$ in Table I) corrects the redistribution below $y^{+}=50$, but amplifies it in the logarithmic layer, as predicted by the theory. Since the source term overestimates the redistribution, formulations for which the theory predicts an amplification factor $\Gamma$ less than one are expected to give more satisfactory results. It can be seen in Fig. 1(a) that the $W 2$ and $M$ models, the latter with $\beta=0.25$, give better results than the original one. The $M$ model slightly better predicts the redistribution below $y^{+} \simeq 150$, but still overestimates it in the rest of the logarithmic layer.

Similar a priori tests have been performed with the SSG model as the source term. Results are shown in Fig. 1(b). It can be seen that the SSG model, without the elliptic relaxation correction, predicts correctly $\phi_{22}^{*}$ in the logarithmic layer. Therefore, it is expected that a neutral model $(\Gamma=1)$ will give better results associated with this source term. It can be seen in Fig. 1(b) that the neutral formulation $W 1$ indeed gives good predictions. However, it induces a slight reduction of $\phi_{22}^{*}$ in the logarithmic layer: this gap between theory and numerical results could be expected in so far as the theory is not strictly valid at this Reynolds number. As regards the $M$ formulation, the deviation from the theoretical result is surprisingly more significant. Indeed, it has appeared that with the theoretical neutral value of the coefficient $(\beta=1 / 12)$, this model still induces an amplification of
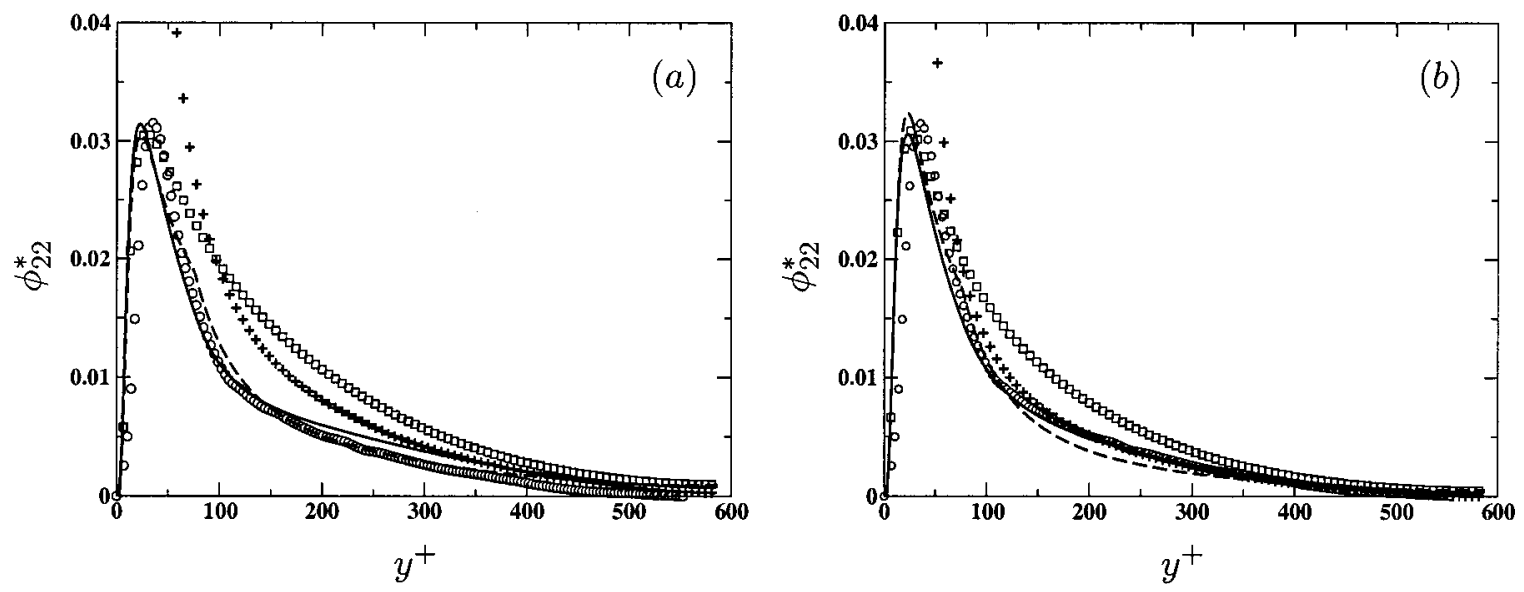

FIG. 1. A priori tests in a channel flow at $\operatorname{Re}_{\tau}=590$. (a) Tests with the Rotta + IP model as the source term: + , source term $\phi_{22}^{h}$ without using the elliptic relaxation equation; $\bigcirc, \phi_{22}^{*}$ from the DNS; $\square$, original model $(D) ;---$, Wizman et al. second model (W2); —, Manceau et al. model with $\beta=0.25$. (b) Tests with the SSG model as the source term: + , source term $\phi_{22}^{h}$ without using the elliptic relaxation equation; $\bigcirc, \phi_{22}^{*}$ from the DNS; $\square$, original model $(D) ;---$, Wizman et al. neutral model $(W 1) ;-$, Manceau et al. model $(M)$ with $\beta=0.17$. 

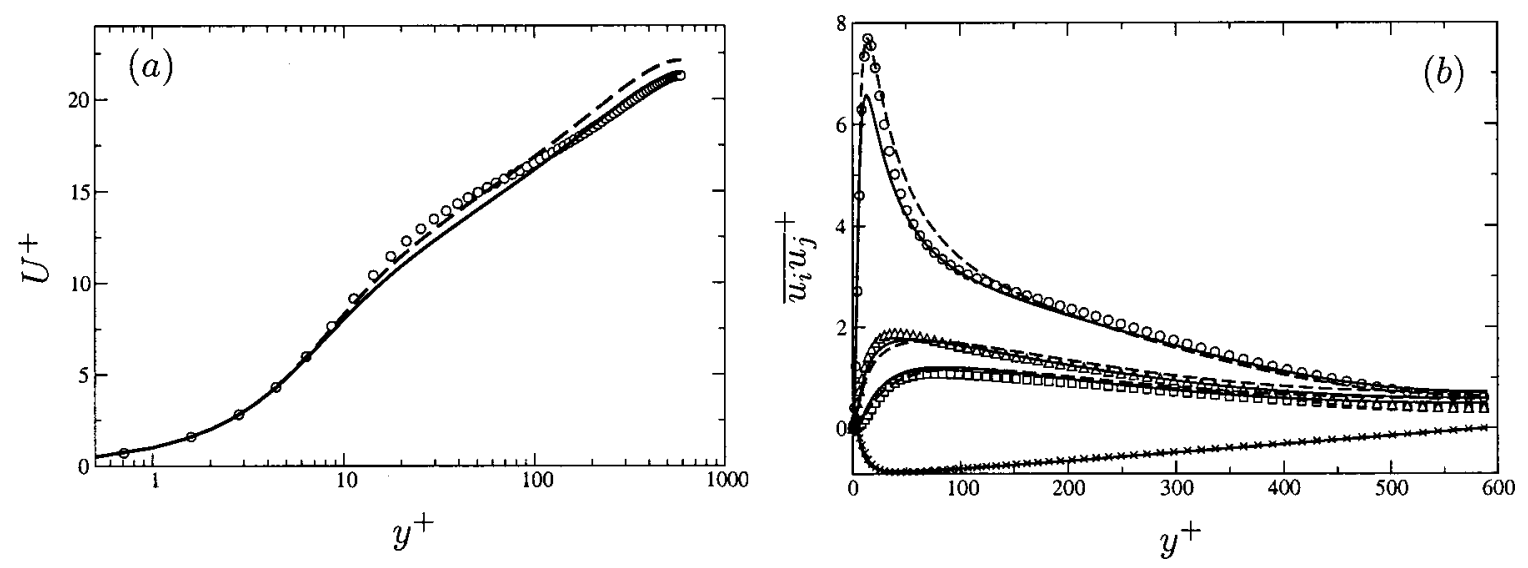

FIG. 2. Test of the influence of varying the coefficient $C_{L}$ with the original formulation ( $D$ model). (a) Velocity profile: $\bigcirc$, DNS;,$- C_{L}=0.2 ;---$, $C_{L}=0.27$. (b) Reynolds stresses. Symbols: DNS $\left(\bigcirc \overline{u^{2}} ; \square \overline{v^{2}} ; \triangle \overline{w^{2}} ; * \overline{u v}\right) ;-, C_{L}=0.2 ;---, C_{L}=0.27$.

the redistribution in the logarithmic layer; the coefficient that gives the best prediction, shown in Fig. 1(b), is actually $\beta=0.17$, i.e., twice the theoretical neutral value. However, results from a priori tests must always be treated with caution, and full tests must be performed to investigate, in particular, the value of $\beta$ to be chosen.

Nevertheless, these tests give some interesting indications about the behavior of the different formulations. It appears that the SSG model (which gives a reasonably correct prediction of the redistribution in the logarithmic layer, and had been widely applied with success in various complex flows) is a better source term for elliptic relaxation models than the Rotta+IP model. Accordingly, the next section will be dedicated to tests of full Reynolds stress models using the SSG model as the source term and neutral formulations of the elliptic operator.

\section{FULL REYNOLDS STRESS MODEL}

In this section, simulations are performed with a full Reynolds stress model, in the same case of the channel flow at $\operatorname{Re}_{\tau}=590$. The equations of the model are given in Appendix A. The SSG model is used as the source term $\phi_{i j}^{h}$. Note that the quadratic slow term $C_{2} k \operatorname{dev}\left(b_{i k} b_{k j}\right) / T$ has been kept in the model, even though it is known to only slightly improve the predictions and to make the model stiff in computations. Following Durbin, ${ }^{6}$ a part of the dissipation, $\varepsilon_{i j}$ $-\overline{u_{i} u_{j}} \varepsilon / k$, is included in $k f_{i j}$. This leads to the appearance of $\overline{u_{i} u_{j}} / k T-2 / 3 \delta_{i j} / T$ in the source term of the elliptic relaxation Eq. (A2) and to the modification of the boundary conditions for $f_{i j}$. Indeed, they are chosen such that $k f_{i j}$ balances the difference between the dissipation $\varepsilon_{i j}$ and the molecular diffusion $D_{i j}^{\nu}$ in the vicinity of the wall. Since $\underline{\varepsilon_{i j}-}-\overline{u_{i} u_{j}} \varepsilon / k$ is included in $k f_{i j}$, the latter must balance $\overline{u_{i} u_{j}} \varepsilon / k-D_{i j}^{\nu}$, which can be achieved $d^{4,18}$ by using the boundary conditions $f_{11}=0, f_{22}=-20 \nu^{2} \overline{v^{2}} / \varepsilon y^{4}, f_{33}=0$, and $f_{12}=-20 \nu^{2} \overline{u v} / \varepsilon y^{4}$. Note that the boundary condition used here for $f_{22}$ is different from the exact one (used in a priori tests), which can be expressed as $f_{22}^{\mathrm{DNS}}=-8 \nu^{2} \overline{v^{2}} / \varepsilon y^{4}$. This difference originates from the fact that $\overline{u_{i} u_{j}} \varepsilon / k$ does not reproduce exactly the correct behavior of $\varepsilon_{i j}$ in the vicinity of the wall.

The aim of this section is to investigate the influence of the form of the elliptic relaxation equation. The problem is solved by a simple 1D code, using finite difference approximation. The equations are solved by imposing the friction velocity $u_{\tau}$. First, the original form of the model, with the coefficients used by Wizman et al.,${ }^{14}$ is tested. It can be seen in Figs. 2(a) and 2(b) that this model reproduces accurately the logarithmic layer, but not the buffer layer. Figure 2(a) shows that the mean velocity in the buffer layer is underestimated, and Fig. 2(b) that the peak of $\overline{u^{2}}$ is too low. This problem can only be solved by increasing the coefficient $C_{L}$, which induces a decrease of the redistribution in the buffer layer. The drawback is the deterioration of the prediction of the mean velocity in the logarithmic layer.

Figures 3(a) and 3(b) show that with the neutral formulations of the elliptic relaxation equation, namely the $W 1$ model and the $M$ model with $\beta=1 / 12$, the peak of $\overline{u^{2}}$ can be correctly reproduced by increasing the coefficient $C_{L}$, without spoiling the mean velocity logarithmic profile. This enables the correct prediction of the mean velocity in both the buffer layer and the logarithmic layer. Note that, contrary to the $a$ priori tests, the full computation with the $M$ model has been performed with the theoretical neutral coefficient $\beta=1 / 12$. In this case, it gives a solution almost indistinguishable from the one given by the $W 1$ model.

As regards the numerical stability, the neutral formulations appear slightly more stable than the original one. For instance, initializing the simulation by coarse empirical formulas, the more stable formulation is the $W 1$ one that admits time steps up to $\Delta t^{+}=15$ without diverging. The upper limit is $\Delta t^{+}=12$ for the $M$ formulation and $\Delta t^{+}=9$ for the original $(D)$ one.

It can be concluded that the use of neutral models leads to significant improvements of the predictions with the SSG model as the source term. Such improvements had already been reported by Wizman et al. ${ }^{14}$ but the model $W 1$ they proposed has been frequently criticized because of its totally 

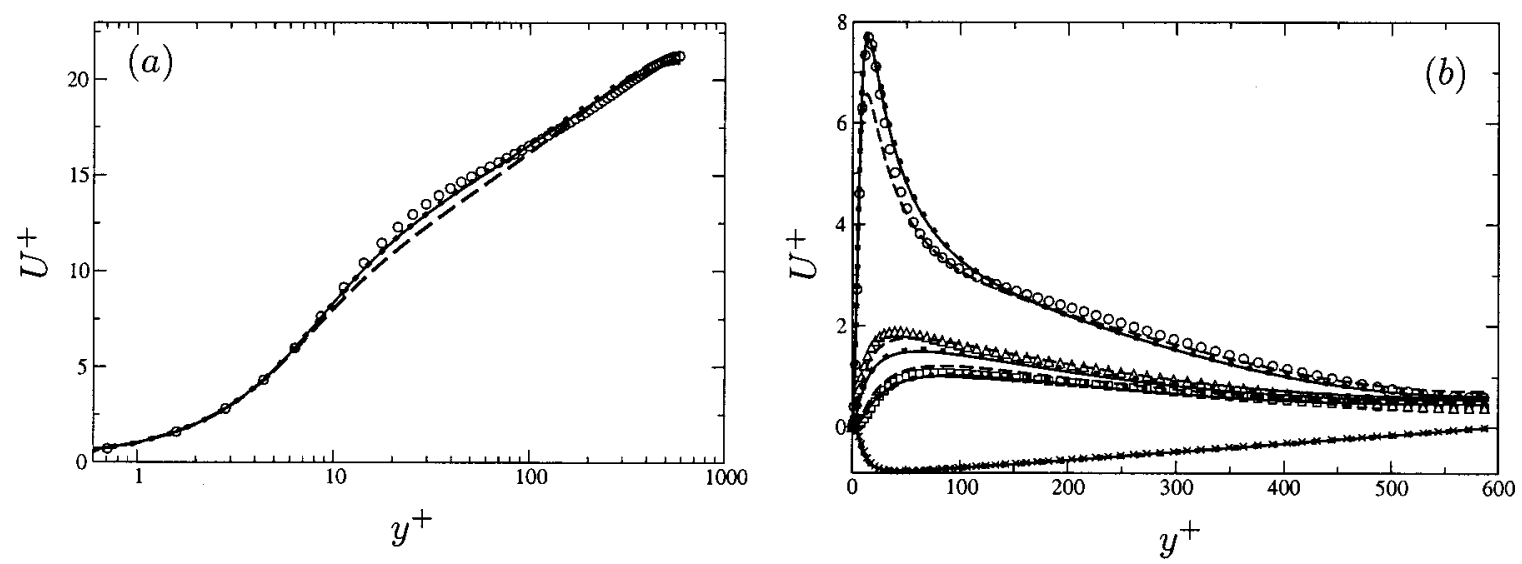

FIG. 3. Improvement obtained with the neutral formulations. (a) Velocity profile: $\bigcirc$, DNS; --- , Original model $(D)$; - , Wizman et al. neutral model (W1); ๑, Manceau et al. neutral model $(M$ with $\beta=1 / 12)$. (b) Reynolds stresses. Symbols: DNS $\left(\bigcirc \overline{u^{2}} ; \square \overline{v^{2}} ; \triangle \overline{w^{2}} ; * \overline{u v}\right)$; --- , Original model; $-\frac{-}{-}$ Wizman et al. neutral model ( $\left.W_{1}\right) ; \boldsymbol{\bullet}$, Manceau et al. neutral model ( $M$ with $\beta=1 / 12$ ).

arbitrary character. On the contrary, the $M$ model is derived from an assumption on the shape of the correlation function introduced to account for physical phenomena observed in DNS data. Even if some approximations have been also made, such as the truncation to the first order of the Taylor series expansion, the model is thus directly related to a theoretical analysis.

\section{IV. $\overline{v^{2}}-f$ MODEL}

In this section, the $\overline{v^{2}}-f$ model will be in focus. Indeed, even though this model has led to satisfactory results in a number of different complex flows, the prediction of the mean velocity profile in a channel is still improvable.

In the frame of an eddy viscosity model, the SSG cannot be chosen as the source term, since it involves invariants of the anisotropy tensor. Therefore, the Rotta+IP model is used. The equations of the $\overline{v^{2}}-f$ model are given in Appendix B, with the coefficients used by Manceau et al. ${ }^{19}$ It can be seen that the coefficients of the transport equation of the dissipation are slightly different from those of the standard $k-\epsilon$ model, and that the coefficients of the Rotta + IP model have also been modified. However, these coefficients have led to satisfactory results in many situations and the analysis of their influence is beyond the scope of this article, which mainly focuses on the influence of the elliptic relaxation operator.

Results given by the original model, with the formulation of the elliptic relaxation equation denoted by $D$ in Table I, are shown in Figs. 4(a) and 4(b). It can be seen that at a given $\operatorname{Re}_{\tau}$, the mean velocity is overestimated in the logarithmic layer. As regards the turbulent kinetic energy, it is slightly overpredicted between $y^{+} \simeq 30$ and $y^{+} \simeq 150$, and underpredicted between $y^{+} \simeq 150$ and $y^{+} \simeq 400$. The velocity scale $\overline{v^{2}}$, which is equivalent to the wall-normal Reynolds stress component in a channel, follows an opposite behavior.

The use of the neutral formulations $W 1$ and $M$, with $\beta$ $=1 / 12$, which are again almost indistinguishable from each other, enables the correction of the mean velocity profile in the logarithmic layer, whereas the prediction of turbulent quantities $k$ and $\overline{v^{2}}$ are not significantly improved. In the first case (model $W 1$ ), no coefficient has been changed, whereas in the second case (model $M$ ) , $C_{L}$ has been slightly in-
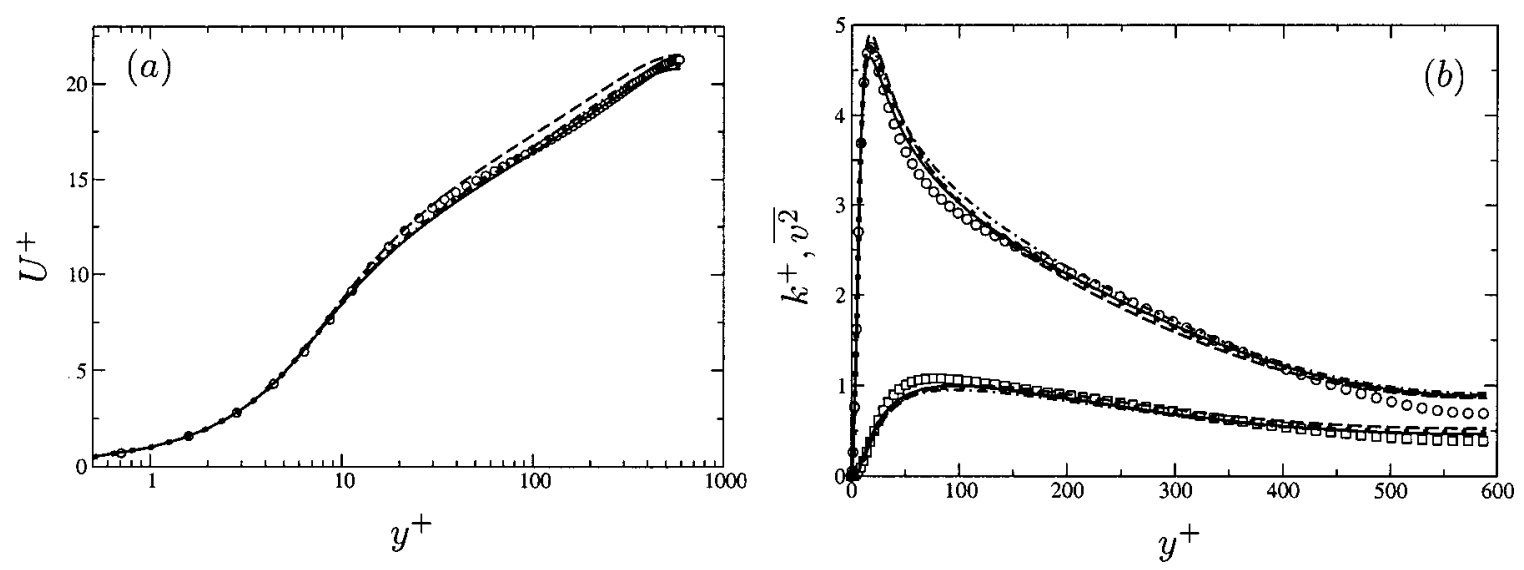

FIG. 4. $\overline{v^{2}}-f$ model. Comparison of the results given by the original formulation and the neutral or reducing ones. (a) Velocity profile: $\bigcirc$, DNS; --- , Original model $(D) ;-$, Wizman et al. neutral model $\left(W_{1}\right) ; \bullet$, Manceau et al. neutral model $(M$ with $\beta=1 / 12) ;-\cdot-\cdot-\cdot-$, Manceau et al. reducing model $(M$ with $\beta=2 / 12)$. (b) Turbulent energy and normal stress. Symbols: DNS $\left(\bigcirc k\right.$; $\left.\square \overline{v^{2}}\right) ;---$, Original model $(D)$; - , Wizman $e t$ al. neutral model $\left(W_{1}\right) ; \bullet$, Manceau et al. neutral model $(M$ with $\beta=1 / 12) ;-\cdot-\cdot-\cdot-$, Manceau et al. reducing model $(M$ with $\beta=2 / 12)$. 
creased. Other coefficients are left unchanged in both cases. In this case, since the Rotta+IP model predicts a too high level of redistribution, it makes sense to use the $W 2$ formulation that exhibits an amplification factor less than one $(\Gamma$ $=0.75$ ), or the $M$ formulation with a coefficient $\beta$ larger than $1 / 12$. Results given by the latter with $\beta=2 / 12$, corresponding to $\Gamma=0.75$, are also shown in Figs. 4(a) and 4(b). The prediction of $k$ in the logarithmic layer is slightly improved compared with neutral formulations, without any significant effect on the velocity profile. Note that the results given by this formulation and those given by the $W 2$ formulation (not shown here) are indistinguishable.

The same stability tests as performed in Sec. III lead to the following upper limits for the time step: $\Delta t^{+}=430$ for $M$ with $\beta=2 / 12, \Delta t^{+}=410$ for $W 1, \Delta t^{+}=380$ for $M$ with $\beta$ $=1 / 12$, and $\Delta t^{+}=365$ for $D$. It appears that the smaller the amplification factor $\Gamma$, the more stable the model. (However, the predictions begin to deteriorate below $\Gamma=0.75$.) Note also that the $\overline{v^{2}}-f$ model is much more stable than the full Reynolds stress model.

The results obtained in this section show that the spurious behavior of the original elliptic operator in the logarithmic layer leads to difficulties in correctly reproducing both the friction velocity and the flow rate. The use of the neutral or reducing formulations overcomes this problem, and also stabilizes the simulation. Again, the $M$ formulation is preferable to the $W 1$ or $W 2$ ones that have been introduced arbitrarily.

\section{CONCLUSION}

The elliptic relaxation method and the problems associated with it in the reproduction of the redistribution in the logarithmic layer have been presented. It has been shown that the amplification of the redistribution can be avoided by introducing the formulation of the elliptic relaxation equation proposed by Manceau et al. ${ }^{15}$ which accounts for the asymmetry of the two-point correlations due to the strong inhomogeneity in the vicinity of the wall.

The behavior of the elliptic operator in the logarithmic region predicted by the theory has first been confirmed by $a$ priori tests in a channel flow at $\operatorname{Re}_{\tau}=590$. The formulation of the operator that gives the best results depends on the quasihomogeneous model used as the source term of the elliptic relaxation equation. With the Rotta+IP model, which overestimates the redistribution in the logarithmic layer, formulations that exhibit an amplification factor less than one are preferable: this is the case, in particular, of the model proposed by Manceau et al., provided that the $\beta$ coefficient is taken larger than $1 / 12$. With the SSG model, which better predicts the redistribution in the logarithmic layer, it is confirmed that neutral models are preferable, even if the results do not exactly conform with the theory.

Simulations with Durbin's full Reynolds stress model have been performed in the same flow with the SSG model as the source term. The original model does not allow the simultaneous correct predictions of the buffer and the logarithmic layer. This problem is overcome by the use of neutral formulations, such as the one proposed by Wizman et al., on an empirical basis, or the one proposed by Manceau et al., with $\beta=1 / 12$. This neutral formulations also have the advantage of increasing the stability of the computations.

Finally, different formulations have been tested in the frame of the $\overline{v^{2}}-f$ model. The original one, which has been successfully applied in a number of complex flows, does not perfectly predict the mean velocity profile in the logarithmic layer of a channel flow. Again, it has been shown that this problem is related to the erroneous amplification of the redistribution. The neutral formulations, as well as formulations inducing a reduction of the redistribution, lead to a significant improvement of the predictions, and these are more stable than the original one.

It can be concluded that in general, either with the full Reynolds stress model or with the $\overline{v^{2}}-f$, neutral formulations are preferable. Similar conclusions have already been drawn by Wizman et al., but their neutral model is seldom used because of its totally empirical foundation. On the contrary, the formulation proposed by Manceau et al. has been derived from the analysis of the two-point correlations, and gives almost exactly the same results as those obtain with the Wizman et al. formulation. The results presented in the present article are encouraging: they lead to the hope that the modified elliptic relaxation models can be applied to more complex flows with some improvement of the predictions.

\section{APPENDIX A: DURBIN'S FULL REYNOLDS STRESS MODEL}

$$
\begin{aligned}
\frac{D \overline{u_{i} u_{j}}}{D t}= & -\overline{u_{i} u_{k}} \frac{\partial U_{j}}{\partial x_{k}}-\overline{u_{j} u_{k}} \frac{\partial U_{i}}{\partial x_{k}}+k f_{i j}-\frac{\overline{u_{i} u_{j}}}{k} \varepsilon \\
& +\frac{\partial}{\partial x_{l}}\left(\frac{C_{\mu}}{\sigma_{k}} \overline{u_{l} u_{m}} T \frac{\partial \overline{u_{i} u_{j}}}{\partial x_{m}}\right)+\nu \nabla^{2} \overline{u_{i} u_{j}}, \\
\mathcal{L}\left(f_{i j}\right)= & \frac{1}{k}\left(\phi_{i j}^{h}-\frac{2}{3} \frac{k}{T} \delta_{i j}+\frac{\overline{u_{i} u_{j}}}{T}\right),
\end{aligned}
$$

with $\mathcal{L}\left(f_{i j}\right)=f_{i j}-L^{2} \nabla^{2} f_{i j} \quad \operatorname{Durbin}^{6}(D)$,

or $\mathcal{L}\left(f_{i j}\right)=f_{i j}-\nabla^{2}\left(L^{2} f_{i j}\right)$, Wizman et al. ${ }^{14}(W 1)$,

or $\mathcal{L}\left(f_{i j}\right)=\left(1+16 \beta(\nabla L)^{2}\right) f_{i j}-L^{2} \nabla^{2} f_{i j}-8 \beta L \nabla L \cdot \nabla f_{i j}$,

$$
\begin{aligned}
& \text { Manceau et al. }{ }^{15}(M) . \\
\phi_{i j}^{h}= & -\left(C_{1} \frac{k}{T}+C_{1}^{*} P\right) b_{i j}+C_{2} \frac{k}{T} \operatorname{dev}\left(b_{i k} b_{k j}\right) \\
& +\left(C_{3}-C_{3}^{*}\left(b_{k l} b_{k l}\right)^{1 / 2}\right) k S_{i j} \\
& +C_{4} k \operatorname{dev}\left(b_{i k} S_{k j}+b_{j k} S_{k i}\right) \\
& +C_{5} k\left(b_{i k} \Omega_{k j}+b_{j k} \Omega_{k i}\right),
\end{aligned}
$$

where

$$
b_{i j}=\frac{\overline{u_{i} u_{j}}}{2 k}-\frac{1}{3} \delta_{i j}, \quad S_{i j}=\frac{1}{2}\left(\frac{\partial U_{i}}{\partial x_{j}}+\frac{\partial U_{j}}{\partial x_{i}}\right),
$$

and 


$$
\begin{aligned}
& \Omega_{i j}=\frac{1}{2}\left(\frac{\partial U_{i}}{\partial x_{j}}-\frac{\partial U_{i}}{\partial x_{i}}\right) . \\
& \frac{D \varepsilon}{D t}=\frac{C_{\varepsilon_{1}}^{\prime} P-C_{\varepsilon_{2}} \varepsilon}{T}+\frac{\partial}{\partial x_{l}}\left(\frac{C_{\mu}}{\sigma_{\varepsilon}} \overline{u_{l} u_{m}} T \frac{\partial \varepsilon}{\partial x_{m}}\right)+\nu \nabla^{2} \varepsilon,
\end{aligned}
$$

where

$$
\begin{aligned}
& T=\max \left(\frac{k}{\varepsilon}, C_{T}\left(\frac{\nu}{\varepsilon}\right)^{2}\right) ; \\
& L=C_{L} \max \left(\frac{k^{3 / 2}}{\varepsilon}, C_{\eta} \frac{\nu^{3 / 4}}{\varepsilon^{1 / 4}}\right) ; C_{\varepsilon_{1}}^{\prime}=C_{\varepsilon_{1}}\left(1+A_{1} \frac{P}{\varepsilon}\right), \\
& C_{\mu}=0.26 ; \quad \sigma_{k}=1.0 ; \quad C_{\varepsilon_{1}}=1.35, C_{\varepsilon_{2}}=1.83 ; \sigma_{\varepsilon}=1.4, \\
& C_{\eta}=80.0 ; \quad C_{T}=6.0 ; A_{1}=0.1 \\
& C_{1}=3.4 ; \quad C_{1}^{*}=1.8 ; \quad C_{2}=4.2 ; C_{3}=1.3 ; \\
& C_{4}=1.25 ; C_{5}=0.4 . \\
& D \text { formulation: } \quad C_{L}=0.2 ;
\end{aligned}
$$

$W 1$ formulation: $C_{L}=0.29$;

$M$ formulation: $C_{L}=0.28$.

Wall boundary conditions:

$$
\begin{aligned}
& U_{i}=0 ; \quad \overline{u_{i} u_{j}}=0 ; \varepsilon=\frac{2 \nu k}{y^{2}} \\
& f_{11}=0 ; f_{22}=-\frac{20 \nu^{2} \overline{v^{2}}}{\varepsilon y^{4}} ; f_{33}=0 ; f_{12}=-\frac{20 \nu^{2} \overline{u v}}{\varepsilon y^{4}} .
\end{aligned}
$$

\section{APPENDIX B: $\overline{v^{2}}-f$ MODEL EQUATIONS}

$$
\frac{D U}{D t}=-\nabla p+\nabla \cdot\left(\left(\nu+\nu_{T}\right)\left(\nabla U+\nabla^{t} U\right)\right),
$$

with $\nu_{T}=C_{\mu} \overline{v^{2}} T$.

$$
\frac{D k}{D t}=P-\varepsilon+\nabla \cdot\left(\left(\nu+\nu_{T}\right) \nabla k\right),
$$

with $P=2 \nu_{T} S_{i j} S_{i j}$.

$$
\begin{aligned}
& \frac{D_{\varepsilon}}{D t}=\frac{C_{\varepsilon_{1}}^{\prime} P-C_{\varepsilon_{2}} \varepsilon}{T}+\nabla \cdot\left(\left(\nu+\frac{\nu_{T}}{\sigma_{\varepsilon}}\right) \nabla_{\varepsilon}\right), \\
& \frac{D \overline{v^{2}}}{D t}=k f-\frac{\overline{v^{2}}}{k} \varepsilon+\nabla \cdot\left(\left(\nu+\nu_{T}\right) \nabla \overline{v^{2}}\right), \\
& \mathcal{L}(f)=\left(C_{1}-1\right) \frac{\left(2 / 3-\overline{v^{2}} / k\right)}{T}+C_{2} \frac{P}{k},
\end{aligned}
$$

with $\mathcal{L}(f)=f-L^{2} \nabla^{2} f \quad \operatorname{Durbin}^{6}(D)$, or $\mathcal{L}=f-\nabla^{2}\left(L^{2} f\right) \quad$ Wizman et al. ${ }^{14}(W 1)$, or $\mathcal{L}(f)=\left(1+16 \beta(\nabla L)^{2}\right) f-L^{2} \nabla^{2} f-8 \beta L \nabla L \cdot \nabla f$

Manceau et al. ${ }^{15}(M)$.

$$
T=\max \left(\frac{k}{\varepsilon} ; C_{T}\left(\frac{\nu}{\varepsilon}\right)^{1 / 4}\right)
$$

$$
\begin{aligned}
& L=C_{L} \max \left(\frac{k^{3 / 2}}{\varepsilon} ; C_{\eta}\left(\frac{\nu^{3}}{\varepsilon}\right)^{1 / 4}\right) ; \\
& C_{\varepsilon_{1}}^{\prime}=C_{\varepsilon_{1}}\left(1+\alpha\left(\frac{k}{\overline{v^{2}}}\right)^{1 / 2}\right) ; \\
& C_{\mu}=0.22 ; \quad C_{\varepsilon_{1}}=1.4 ; \quad C_{\varepsilon_{2}}=1.9 ; \quad \sigma_{\varepsilon}=1.3 ; \quad C_{1}=1.4 ; \\
& C_{2}=0.3 ; \quad C_{\eta}=85.0 ; \quad C_{T}=6.0 ; \alpha=0.045 . \\
& D \text { formulation: } C_{L}=0.22,
\end{aligned}
$$

$W 1$ formulation: $C_{L}=0.22$,

$M$ formulation: $C_{L}=0.25$.

Wall boundary conditions:

$$
U_{i}=0 ; k=0 ; \quad \overline{v^{2}}=0 ; \quad \varepsilon=\frac{2 \nu k}{y^{2}} ; f=-\frac{20 \nu^{2} \overline{v^{2}}}{\varepsilon y^{4}} .
$$

${ }^{1}$ P. Y. Chou, " On velocity correlations and the solutions of the equations of turbulent fluctuation," Quarterly Appl. Math. 3, 38 (1945).

${ }^{2}$ P. Y. Chou, "Pressure flow of a turbulent fluid between two parallel infinite planes," Quarterly Appl. Math. 3, 198 (1945).

${ }^{3}$ J. C. Rotta, "Statistische Theorie nichthomogener Turbulenz," Z. Phys. 129, 547 (1951).

${ }^{4} R$. Manceau, "Modélisation de la turbulence. Prise en compte de l'influence des parois par relaxation elliptique," Ph.D. thesis, Université de Nantes, 1999.

${ }^{5}$ C. G. Speziale, "A review of Reynolds stress models for turbulent shear flows," Technical Report 95-15, ICASE, NASA, 1995.

${ }^{6} \mathrm{P}$. A. Durbin, "Near-wall turbulence closure modeling without 'damping functions',"' Theor. Comput. Fluid Dyn. 3, 1 (1991).

${ }^{7}$ P. A. Durbin, "A Reynolds stress model for near-wall turbulence," J. Fluid Mech. 249, 465 (1993).

${ }^{8}$ M. Behnia, S. Parneix, and P. Durbin, "Prediction of heat transfer in a jet impinging on a flat plate,' Int. J. Heat Mass Transf. 41, 1845 (1998).

${ }^{9}$ M. Behnia, S. Parneix, Y. Shabany, and P. A. Durbin, "Numerical study of turbulent heat transfer in confined and unconfined impinging jets," Int. J. Heat Fluid Flow 20, 1 (1999).

${ }^{10}$ P. A. Durbin, "Separated flow computations with the $k-\varepsilon-\overline{v^{2}}$ model," AIAA J. 33, 659 (1995).

${ }^{11}$ F.-S. Lien, P. A. Durbin, and S. Parneix, "Non-linear $\overline{v^{2}}-f$ modelling with application to aerodynamic flows," Proceedings of the 11th Symposium on Turbulent Shear Flows, Grenoble, 1997, Vol. 6, pp. 19-24.

${ }^{12}$ S. Parneix, M. Behnia, and P. A. Durbin, "Predictions of turbulent heat transfer in an axisymmetric jet impinging on a heated pedestal," J. Heat Transfer 120, 1 (1998).

${ }^{13}$ S. Parneix, P. A. Durbin, and M. Behnia, "Computation of 3D turbulent boundary layers using the $\overline{v^{2}}-f$ model," Flow, Turb. and Comb. 60, 19 (1998).

${ }^{14}$ V. Wizman, D. Laurence, M. Kanniche, P. Durbin, and A. Demuren, "Modeling near-wall effects in second-moment closures by elliptic relaxation," Int. J. Heat Fluid Flow 17, 255 (1996).

${ }^{15}$ R. Manceau, M. Wang, and D. Laurence, "Inhomogeneity and anisotropy effects on the redistribution term in RANS modeling," J. Fluid Mech. (to be published).

${ }^{16}$ R. Manceau, M. Wang, and P. Durbin, Assessment of Nonlocal Effect on Pressure Term in RANS Modeling Using a DNS Database, Proceedings of the Summer Program, Center for Turbulence Research, Stanford University (1998), pp. 303-322.

${ }^{17}$ R. D. Moser, J. Kim, and N. N. Mansour, "'Direct numerical simulation of turbulent channel flow up to $\mathrm{Re}_{\tau}=590$," Phys. Fluids 11, 943 (1999).

${ }^{18} \mathrm{R}$. Manceau, Reproducing the Blocking Effect of the Wall in One-Point Turbulence Models, Proceedings of the European Congress Comput. Meth. Appl. Sciences and Engineering, Barcelona, 2000, pp. 1-20.

${ }^{19}$ R. Manceau, S. Parneix, and D. Laurence, "Turbulent heat transfer predictions using the $\overline{v^{2}}-f$ model on unstructured meshes," Int. J. Heat Fluid Flow 21, 320 (2000). 